\title{
Proteases of a Bacillus subtilis Clinical Isolate Facilitate Swarming and Siderophore-Mediated Iron Uptake via Proteolytic Cleavage of Transferrin
}

\author{
Ra-Young Park, ${ }^{a}$ Hui-Yu Sun, ${ }^{a}$ Mee-Hwa ChOI,${ }^{a}$ Young-Hoon BAI, ${ }^{b}$ Yoon-Young Chung, ${ }^{c}$ and \\ Sung Heui SHIN $*, a$ \\ ${ }^{a}$ Research Center for Resistant Cells, Chosun University Medical School; ${ }^{b}$ Department of Biology, Chosun University \\ Medical School; and ${ }^{c}$ Department of Anatomy, Chosun University Medical School; Gwangju 501-759, Republic of Korea. \\ Received July 25, 2005; accepted October 24, 2005
}

\begin{abstract}
We isolated a highly serine protease-producing Bacillus subtilis strain (PRY) from a clinical sample and identified it through biochemical testing and ribosomal DNA sequencing. The PRY strain exhibited a robust swarming behavior and was able to digest human transferrin efficiently, concomitantly with the production of catechol-siderophore in the exponential growth phase. The growth of PRY was in proportion to increased iron availability resulting from transferrin destruction. These results suggest that proteases of the B. subtilis PRY strain may play a significant role in the pathogenesis of human infections by facilitating siderophore-mediated iron uptake from transferrin and swarming motility.
\end{abstract}

Key words Bacillus subtilis; protease; transferrin; iron; swarming

Bacillus subtilis is a spore-forming soil bacterium that occasionally causes human infections, which include eye, soft tissue, and lung infections, that are usually associated with immunosuppression, trauma, an indwelling catheter, or contaminination of complex equipment such as an artificial kidney. ${ }^{1)}$ Few studies have examined the virulence factors of B. subtilis because of the relatively few human infections caused by the bacterium.

B. subtilis produces several extracellular proteases including subtilisin. ${ }^{2)}$ In particular, B. subtilis extracellular proteases have recently been found to be essential for swarming motility. ${ }^{2,3)}$ Bacterial swarming is defined as a rapid and coordinated population migration of flagellated bacteria across solid surfaces and is known to be a good in vitro model of bacterial surface adherence and colonization. ${ }^{4,5}$ Moreover, bacterial swarming is coupled to the expression of proteases in other bacteria and closely linked with biofilm formation, antibiotic resistance, and virulence factor production, to the extent that it is itself considered an important virulence factor. $^{4-7)}$

In addition to the association with swarming, it has been suggested that extracellular proteases can augment siderophore-mediated iron uptake from transferrin via the proteolytic cleavage of transferrin in certain bacteria. ${ }^{8-10}$ This proteolytic cleavage of transferrin may facilitate the in vivo growth of protease-producing bacteria and thus may play a significant role in the pathogenesis of human infections caused by such bacteria. As most extracellular iron in the human body is bound to iron-withholding glycoproteins including transferrin, which are considered to be an important constituent of nonspecific innate immunity, bacteria cannot easily acquire iron from transferrin. Accordingly, for certain bacteria to grow and infect humans, they must first acquire iron efficiently from transferrin.

Most pathogenic bacteria have developed specific iron-uptake systems for this specific purpose, and a siderophore-mediated iron-uptake system is a basic and essential system in most bacteria. ${ }^{11)}$ Bacillus species also produce catecholsiderophore and are able to take up iron from transferrin using this siderophore. ${ }^{12,13)}$ However, several studies failed to demonstrate the direct involvement of bacterial proteases in facilitating the siderophore-mediated iron uptake from transferrin, because most bacterial proteases are produced during the late growth phase when most iron has already been consumed. ${ }^{6,14,15)}$ Accordingly, for proteases to augment siderophore-mediated iron uptake from transferrin in bacteria, sufficient proteases capable of destroying transferrin must be produced during the exponential growth phase concomitantly with the production of siderophores.

We have recently isolated a highly protease-producing $B$. subtilis strain (PRY) from wounds on the legs of a traffic accident patient. The wounds were infected with mixed bacteria including the B. subtilis strain. We found that the serine proteases produced by the PRY strain were able to facilitate both the swarming motility and the siderophore-mediated iron uptake from transferrin via the destruction of transferrin. These findings suggest that the proteases of the B. subtilis PRY strain may play a significant role in the pathogenesis of human infections by facilitating the siderophore-mediated iron uptake from transferrin and the swarming motility.

\section{MATERIALS AND METHODS}

Bacterial Strains The PRY strain used in this study exhibited remarkably large clear zones around colonies on skim milk agar (data not shown). The strain was identified by biochemical testing and $16 \mathrm{~s}$ ribosomal DNA sequencing, which were performed by the Korean Culture Center of Microorganisms (KCCM, http://www.kccm.or.kr). Biochemical testing using the API $50 \mathrm{CHB}$ kit (BioMerieux) revealed an $88.2 \%$ identification $(\mathrm{T}$ index $=0.94)$ for $B$. subtilis. Ribosomal DNA sequencing using a universal pair of primers (5'-AGAGTTTGATCATGGCTCAG-3' and 5'-GGATACCTTGTTACGACTT- $3^{\prime}$ ) revealed $99 \%$ homology to B. subtilis strain DSM10 (accession no. AJ276351). The domestic B. subtilis ATCC 9372 (KCCM 11314) strain was purchased from the KCCM and used as a control strain, and its extracellular protease activity on skim milk agar was markedly lower 
than that of the PRY strain (data not shown).

Protease Assays The B. subtilis ATCC 9372 and PRY strains grown in Brain Heart Infusion (BHI, Difco) broths at $37^{\circ} \mathrm{C}$ for $12 \mathrm{~h}$ were inoculated into MM9 minimal broths ${ }^{16)}$ at a concentration of $1 \times 10^{6}$ colony-forming units $(\mathrm{cfu}) / \mathrm{ml}$, and cultured with vigorous shaking $(220 \mathrm{rpm})$ at $37^{\circ} \mathrm{C}$ for $24 \mathrm{~h}$. Culture supernatants were obtained at appropriate times by centrifugation $(10000 \mathrm{rpm}, 5 \mathrm{~min})$. To compare the total protease production between the two strains, we measured caseinolytic activity in the culture supernatants in accordance with the method described in our previous study. ${ }^{17)}$ To compare the proteolytic activity of proteases between the two strains, we performed zymography. After determining the total protein concentration of the culture supernatants obtained at $12 \mathrm{~h}$, the same amounts $(10 \mu \mathrm{g})$ of proteins were electrophoresed without heating on SDS-polyacrylamide gel $(12 \%)$ containing $0.3 \%$ skim milk or human holotransferrin (HT; $1200-1600 \mu \mathrm{g}$ of iron per $1 \mathrm{~g}$ of the protein; Sigma). Other procedures for zymography were performed in accordance with the methods described previously. ${ }^{18)}$ The background of gel was stained with Coomassie blue. For partial characterization of the proteases, we added phenylmethylsulfonylfluoride (PMSF; $10 \mathrm{~mm}$ ) to the culture supernatant from the PRY strain, allowed the mixture to react for $30 \mathrm{~min}$, and then electrophoresed it without heating on SDS-polyacrylamide gel containing $0.3 \%$ skim milk or HT for zymography. To visualize proteolytic cleavage by the proteases of the PRY strain, we added PBS or PMSF (10 mM) to the culture supernatants containing $0.5 \mathrm{mg} / \mathrm{ml}$ of bovine serum albumin (BSA; Sigma) or HT, allowed the mixture to react for $30 \mathrm{~min}$, and then electrophoresed it with heating on ordinary SDSpolyacrylamide gel. The gel was stained with Coomassie blue.

Observation of Swarming To compare swarming motility, the two strains grown in BHI broth at $37^{\circ} \mathrm{C}$ for $12 \mathrm{~h}$ were resuspended at a concentration of $1 \times 10^{7} \mathrm{cfu} / \mathrm{ml}$ in fresh MM9 broths and inoculated onto the surface of MM9 semisolid agars containing $0.7 \%$ agar using blunt-ended toothpicks. To observe the inhibitory effects of the serine protease inhibitor PMSF on the swarming motility of the PRY strain, the strain was inoculated onto the surface of BHI semisolid agars $(0.7 \%$ agar) containing PMSF 10 and $20 \mathrm{~mm}$. The agar plates were incubated at $37^{\circ} \mathrm{C}$ for $24 \mathrm{~h}$.

Observation of Bacterial Growth, Destruction of Transferrins, and Siderophore Production The two strains grown in $\mathrm{BHI}$ broth at $37^{\circ} \mathrm{C}$ for $12 \mathrm{~h}$ were inoculated into MM9 broth containing $0.5 \mathrm{mg} / \mathrm{ml}$ of apotransferrin (AT; less than $30 \mu \mathrm{g}$ of iron per $1 \mathrm{~g}$ of the protein; Sigma), partially iron-saturated transferrin (PT; $300-600 \mu \mathrm{g} / \mathrm{ml}$ of iron per $1 \mathrm{~g}$ of the protein; Sigma), or HT to $1 \times 10^{6} \mathrm{cfu} / \mathrm{ml}$, and cultured with vigorous shaking $(220 \mathrm{rpm})$ at $37^{\circ} \mathrm{C}$ for $24 \mathrm{~h}$. During culture, culture aliquots were withdrawn at appropriate times. Bacterial growth was monitored by measuring the optical density at $600 \mathrm{~nm}\left(\mathrm{OD}_{600}\right)$ of the culture aliquots. Culture supernatants were obtained by centrifuging $(10000 \mathrm{rpm}, 5 \mathrm{~min})$ the culture aliquots. Catecholsiderophore levels in the culture supernatants were measured using the Arnow test. ${ }^{19)}$ The destruction of transferrin in the culture supernatants was visualized with SDS-polyacylamide gel electrophoresis (PAGE) and Coomassie blue staining.

\section{RESULTS AND DISCUSSION}

PRY Produced High Levels of Serine Proteases The production of proteases by the two strains $B$. subtilis ATCC 9372 and PRY was compared using three assay methods. When total protease production was quantitatively compared on a time scale by measuring the caseinolytic activity in the culture supernatants, the PRY strain produced more proteases throughout the culture than the control domestic strain (Fig. 1A). When the proteolytic activity of the two strains was compared on zymogram containing skim milk and on SDSPAGE, the PRY strain also showed much stronger proteolytic activity than the control domestic strain (Figs. 1B, C). Similar results with minor variation in types of proteases were observed when BSA or gelatin was used as a substrate for proteases (data not shown). Accordingly, the PRY strain appeared to produce all proteases at higher levels than the control strain. The proteolytic activity of the PRY strain on both zymography and SDS-PAGE was inhibited by the addition of PMSF $10 \mathrm{~mm}$ (Figs. 1B, C), which indicated that the activity was due to serine proteases.
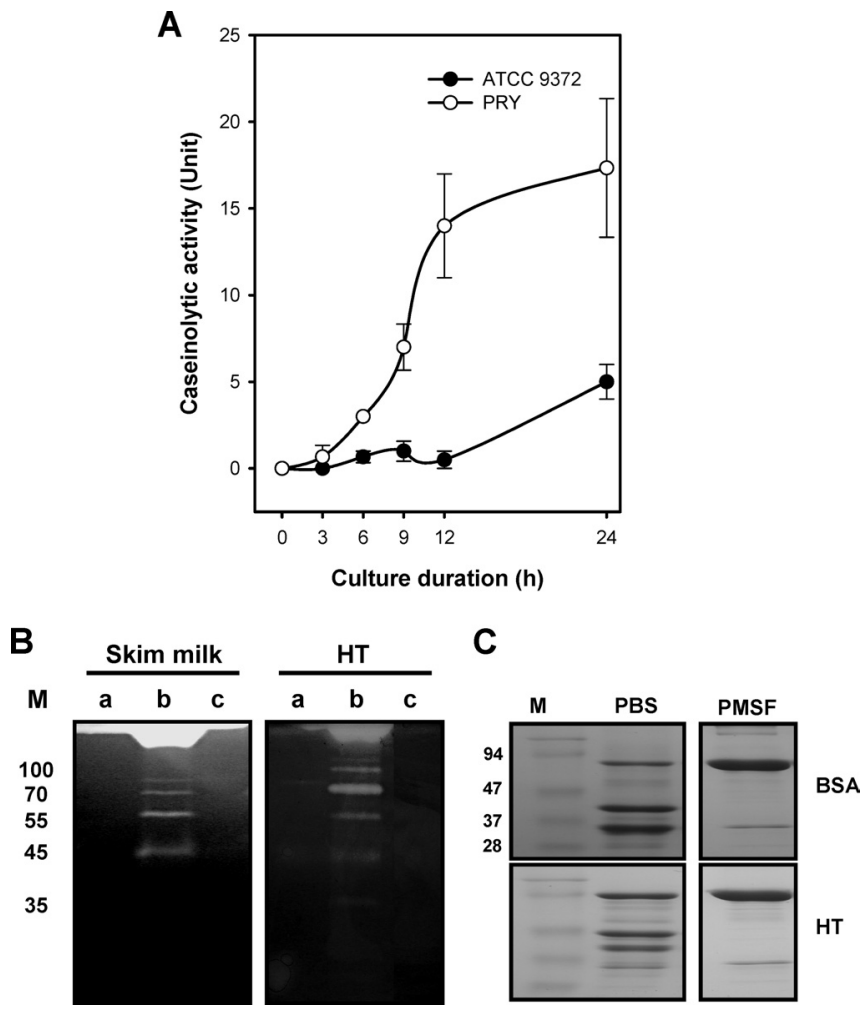

Fig. 1. Comparison of Protease Production

The Bacillus subtilis ATCC 9372 and PRY strains were cultured at $37^{\circ} \mathrm{C}$ for $24 \mathrm{~h}$. Culture supernatants were obtained at the indicated times by centrifuging culture aliquots. (A) Total protease production by B. subtilis ATCC 9372 and PRY strains. Total protease production was compared on a time scale base on the caseinolytic activity in the culture supernatants. (B) Proteolytic activity and its inhibition by PMSF on zymography. After total proteins in the culture supernatants obtained at $12 \mathrm{~h}$ from the ATCC 9372 (a) and PRY (b) strains were quantified, the culture supernatants containing the same amount $(10 \mu \mathrm{g})$ of total proteins were electrophoresed on SDS-polyacrylamide gel containing $0.3 \%$ skim milk and holotransferrin (HT). To observe the effect of PMSF (c) on the proteolytic activity, the culture supernatant from the PRY strain was treated with PMSF $10 \mathrm{~mm}$ at $37^{\circ} \mathrm{C}$ for $30 \mathrm{~min}$ and then electrophoresed on the same gels. The gels were stained with Coomassie blue. (C) Proteolytic cleavage of BSA or HT and its inhibition by PMSF. The culture supernatant (20 $\mu \mathrm{l})$ from the PRY strain at $12 \mathrm{~h}$ was mixed with HT $(0.5 \mathrm{mg} / \mathrm{ml})$ or bovine serum albumin (BSA, $0.5 \mathrm{mg} / \mathrm{ml}$ ), and PBS or PMSF $(10 \mathrm{~mm})$. The mixtures were allowed to react for $30 \mathrm{~min}$, and then routine SDS-PAGE was performed. 
Proteases Are Required for Expression of the Swarming Phenotype of $\boldsymbol{B}$. subtilis Strain PRY Whereas the control strain showed no swarming motility on the surface of MM9 agars containing various concentrations of agar, the PRY strain exhibited obvious swarming motility that was inversely proportional to the viscosity of the agar (Fig. 2A). The swarming of the PRY strain was dose-dependently inhibited by PMSF (Fig. 2B). These results indicate that the extracellular serine proteases are involved in the expression of the swarming phenotype of the B. subtilis PRY strain. Laboratory domestic strains are known to be inherently defective in the expression of swarming, ${ }^{20,21)}$ and this was also true for the control strain used in this study. Our results suggest that the defect in the swarming motility of such laboratory domestic strains may be due to a defect in protease production. Recent studies have revealed that extracellular proteolytic activity could play a central role in swarming motility in $B$. subtilis, ${ }^{2,3)}$ and the production of proteases was stimulated more in swarming bacterial cells than in planktonic bacterial cells. ${ }^{4,5)}$ The swarming motility of $B$. subtilis is associated with total proteolytic activity rather than the activity of a specific protease and is inhibited by $\mathrm{PMSF}^{2}$ ) Such results are consistent with our results. As bacterial swarming itself is considered to be an important virulence factor, ${ }^{5-7)}$ our results suggest that the proteases of the B. subtilis PRY strain may play a significant role in the pathogenesis of human infections via the elicitation of swarming.

Proteases of $B$. subtilis Strain PRY Augment Siderophore-Mediated Iron Uptake via Proteolytic Transfer-

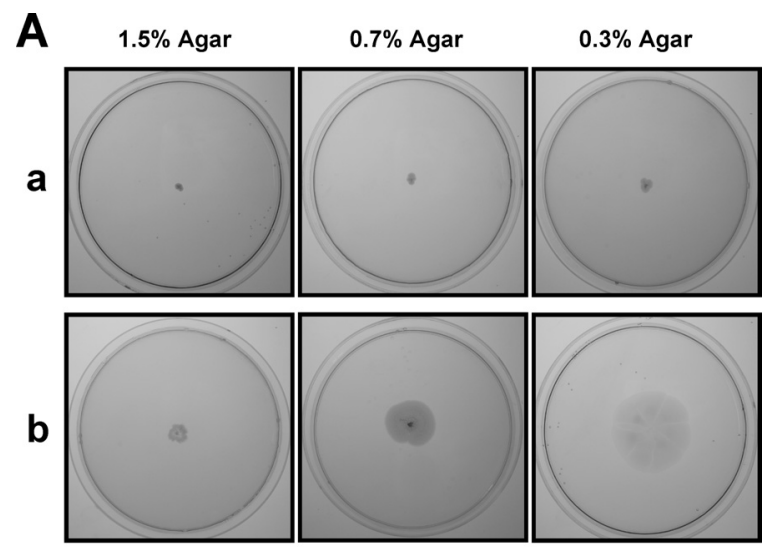

B

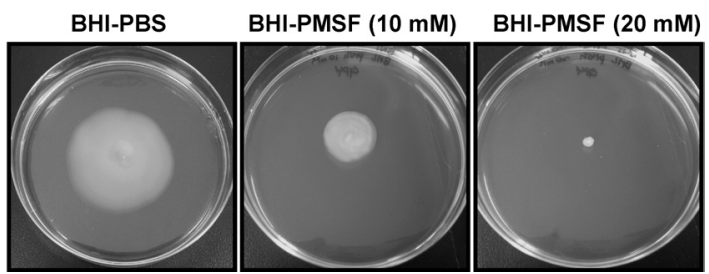

Fig. 2. (A) Swarming Motilities of Bacillus subtilis ATCC 9372 (a) and PRY (b) Strains on MM9 Agars with Various Viscosities

The bacteria grown in BHI broths at $37^{\circ} \mathrm{C}$ for $12 \mathrm{~h}$ were resuspended in fresh MM9 broth to $1 \times 10^{7} \mathrm{cfu} / \mathrm{ml}$ and then inoculated onto the surface of MM9 agars $(0.7 \%$ agar $)$ using blunt-ended toothpicks. The agar plates were incubated at $37^{\circ} \mathrm{C}$ for $24 \mathrm{~h}$.

(B) Inhibition of Swarming Motility of B. subtilis PRY Strain by PMSF.

The PRY strain grown in BHI broths at $37^{\circ} \mathrm{C}$ for $12 \mathrm{~h}$ were resuspended in fresh $\mathrm{BHI}$ broth to $1 \times 10^{7} \mathrm{cfu} / \mathrm{ml}$ and then inoculated onto the surface of BHI agars $(0.7 \%$ agar containing PMSF using blunt-ended toothpicks. The agar plates were incubated at 37 ${ }^{\circ} \mathrm{C}$ for $24 \mathrm{~h}$. rin Cleavage Interestingly, the proteolytic activity of the PRY strain was also observed on a zymogram using $0.3 \%$ human HT (Fig. 1B). Similar results were also observed on zymograms using AT or PT (data not shown). The transferrin-destroying activity was also inhibited by PMSF (Figs. 1B, C), which indicate the role of serine proteases. These findings show that the serine proteases produced by the $B$. subtilis PRY strain can destroy transferrin. However, the destruction of transferrin by proteases alone does not confirm the involvement of the proteases in the facilitation of siderophoremediated iron uptake from transferrin. For proteases to facilitate siderophore-mediated iron uptake from transferrin, the proteolytic cleavage of transferrin must be accompanied by the production of siderophores in the exponential growth phase.

Accordingly, to observe whether proteolytic cleavage of transferrin is accompanied by siderophore production in the exponential growth phase, we cultured the PRY and control strains in MM9 broth containing $0.5 \mathrm{mg} / \mathrm{ml}$ of AT, PT, or HT. The growth of the control strain was found to be correlated with catechol-siderophore production, but not with the ironsaturation level of transferrin. In contrast, the growth of the PRY strain was found to be correlated with the iron-saturation level of transferrin and inversely correlated with catechol-siderophore production (Fig. 3A). The control domestic strain produced more catechol-siderophore than the PRY strain. The destruction of transferrin in the control strain was

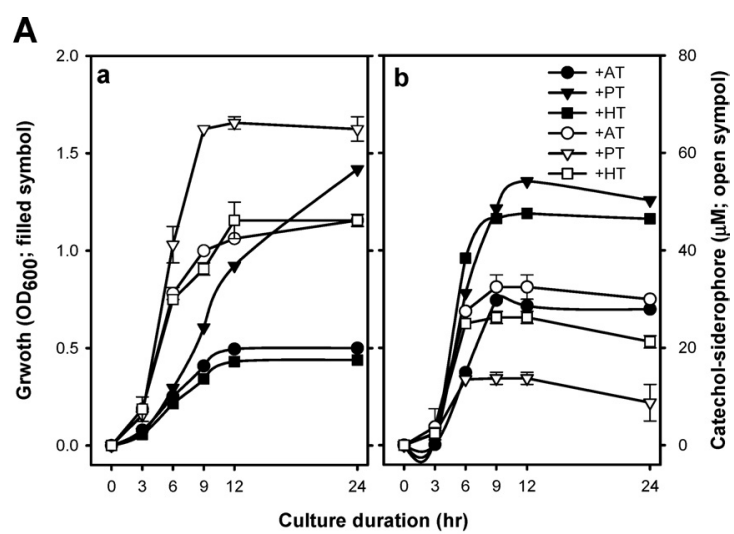

B

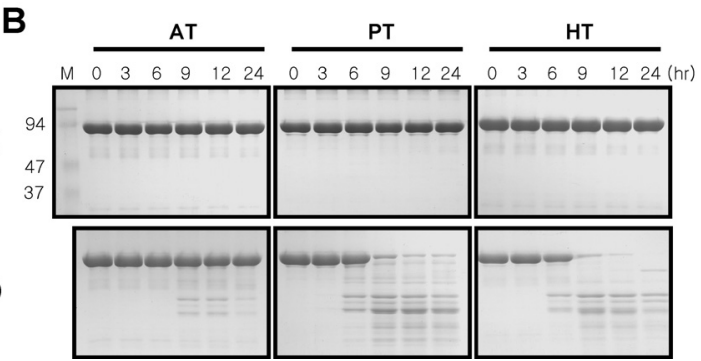

Fig. 3. (A) Growth and Catechol-Siderophore Production, and (B) Transferrin Destruction by Bacillus subtilis ATCC 9372 (a) and PRY (b) Strains in MM9 Broths Containing $0.5 \mathrm{mg} / \mathrm{ml}$ of Apotransferrin (AT), Partially IronSaturated Transferrin (PT), or Holotransferrin (HT)

Bacteria were inoculated into MM9 broths to $1 \times 10^{6} \mathrm{cfu} / \mathrm{ml}$ and cultured with vigorous shaking $(220 \mathrm{rpm})$ at $37^{\circ} \mathrm{C}$ for $24 \mathrm{~h}$. Culture aliquots and culture supernatants were obtained at the indicated times. Bacterial growth was monitored by measuring the $\mathrm{OD}_{600}$ of culture aliquots. The catechol-siderophore level in culture supernatants was measured in triplicate using the Arnow test, and values are expressed as mean and standard errors. Transferrin destruction in the culture supernatants was visualized using routine SDS-PAGE. 
not observed throughout the 24-h culture, whereas that in the PRY strain was initiated at the exponential growth phase (Fig. 3B) and was proportional to bacterial growth and inversely proportional to the production of catecholsiderophore. It is known that the proteolytic cleavage of transferrin by bacterial proteases augments the release of free iron from transferrin and thus elevates iron availability. ${ }^{9)}$ Accordingly, our results indicate that the growth of the control strain was more closely associated with the production of siderophore rather than the iron-saturation level of transferrin because transferrins were not destroyed and freely available iron was not released, whereas the growth of the PRY strain was more closely associated with the iron-saturation level of transferrin rather than the production of catechol-siderophore because the transferrins were destroyed by proteases and freely available iron was released. Bacterial growth is generally dependent on either the production of siderophores or the iron-saturation level of transferrin or iron availability. The production of siderophores is negatively regulated by iron availability via the Fur repressor. ${ }^{11)}$ It is well documented that the production of siderophore by B. subtilis is also regulated by iron availability via the Fur repressor, as in other bacteria. $^{22-24)}$ Our results are consistent with these well-documented concepts.

The involvement of proteases in facilitating the siderophore-mediated iron uptake from transferrin observed in this study is an interesting finding. Recently, we have found that Pseudomonas aeruginosa can also produce proteases capable of destroying transferrin during the exponential growth phase (details will be reported elsewhere), as in this study. In contrast, we also reported that a representative metalloprotease of Vibrio vulnificus could not be directly involved in facilitating the siderophore-mediated iron-uptake from transferrin because it was produced only in the late growth phase and because the inactivation of the protease gene did not affect the siderophore-mediated iron uptake from transferrin. ${ }^{17)}$

In conclusion, the highly serine protease-producing PRY strain exhibited a robust swarming motility and was able to digest human transferrins efficiently, concomitantly with the production of catechol-siderophore in the exponential growth phase. These results suggest that the proteases of the $B$. subtilis PRY strain may play a significant role in the pathogenesis of human infections by facilitating both siderophore-mediated iron uptake from transferrin and swarming motility.
Acknowledgements This study was supported by a grant (R13-2003-009) from the Ministry of Science and Technology, Korea, and the Korean Science and Engineering Foundation through the Research Center for Resistant Cells.

\section{REFERENCES}

1) Drobniewski F. A., Clin. Microbiol. Rev., 6, 324-338 (1993).

2) Connelly M. B., Young G. M., Sloma A., J. Bacteriol., 186, 41594167 (2004).

3) Dixit M., Murudkar C. S., Rao K. K., J. Bacteriol., 184, 596-599 (2002).

4) Givskov M., Eberl L., Molin S., FEMS Microbiol. Lett., 148, 115122 (1997).

5) Walker K. E., Moghaddame-Jafari S., Lockatell C. V., Johnson D., Belas R., Mol. Microbiol., 32, 825-836 (1999).

6) Allison C., Lai H. C., Hughes C., Mol. Microbiol., 6, 1583-1591 (1992).

7) Kim W., Killam T., Sood V., Surette M. G., J. Bacteriol., 185, 31113117 (2003).

8) Britigan B. E., Hayek M. B., Doebbeling B. N., Fick R. B., Jr., Infect. Immun., 61, 5049-5055 (1993).

9) Shigematsu T., Fukushima J., Oyama M., Tsuda M., Kawamoto S., Okuda K., Microbiol. Immunol., 45, 579-590 (2001).

10) Okujo N., Akiyama T., Miyoshi S., Shinoda S., Yamamoto S., Microbiol. Immunol., 40, 595-598 (1998).

11) Ratledge C., Dover L. G., Ann. Rev. Microbiol., 54, 881-941 (2000).

12) May J. J., Wendrich T. M., Marahiel M. A., J. Biol. Chem., 276, 7209-7217 (2001).

13) Park R. Y., Choi M. H., Sun H. Y., Shin S. H., Biol. Pharm. Bull., 28, 1132-1135 (2005).

14) Wolz C., Hohloch K., Ocaktan A., Poole K., Evans R. W., Rochel N., Albrecht-Gary A., Abdallah M. A., Doring G., Infect. Immun., 62, 4021-4027 (1994).

15) Kim S. Y., Lee S. E., Kim Y. R., Kim C. M., Ryu P. Y., Choy H. E., Chung S. S., Rhee J. H., Mol. Microbiol., 48, 1647-1664 (2003).

16) Schwyn B., Neilands J. B., Anal. Biochem., 160, 47-56 (1987).

17) Shin S. H., Sun H. Y., Park R. Y., Kim C. M., Kim S. Y., Rhee J. H., FEMS Microbiol. Lett., 247, 221-229 (2005).

18) Thangam E. B., Rajkumar G. S., Biotechnol. Appl. Biochem., 35, $149-154$ (2002)

19) Arnow L. E., J. Biol. Chem., 118, 531-537 (1937).

20) Harshey R. M., Mol. Microbiol., 13, 389-394 (1994).

21) Kearns D. B., Losick R., Mol. Microbiol., 49, 581-590 (2003).

22) Bsat N., Herbig A., Casillas-Martinez L., Setlow P., Helmann J. D., Mol. Microbiol., 29, 189-198 (1998).

23) Finlay B. B., Falkow S., Microbiol. Mol. Biol. Rev., 61, 136-169 (1997).

24) Harvie D. R., Vilchez S., Steggles J. R., Ellar D. J., Microbiology, 151, $569-577$ (2005). 